\title{
Comportamiento reproductivo en granjas porcinas seropositivas al virus del síndrome reproductivo y respiratorio porcino (VPRRS)
}

\section{Reproductive performance in pig farms seropositive to porcine reproductive and respiratory syndrome virus (VPRRS)}

\author{
Miguel Edmundo Lucho-Cerga ${ }^{1}$, Hilario Noberto Pujada Abad², \\ Felix Esteban Airahuacho-Bautista ${ }^{2}$
}

\section{Resumen}

El presente estudio evaluó la duración de los días de gestación (DG) y el tamaño de camada (TC) en marranas de granja de costa central seropositiva y granja de selva central seronegativa al Virus del Síndrome Reproductivo y Respiratorio Porcino (VPRRS) durante los años 2015 y 2016. Marranas de granja seropositiva a VPRRS muestran DG aumentados y TC disminuidos en granja seropositiva a VPRRS comparado con los de selva seronegativa en 2015. Los DG de marranas de granja seropositiva a VPRRS fueron de menor duración en 2015 en comparación con 2016. La DG y el TC no fueron influenciados por la época de inseminación artificial. Menor DG con mayor TC expresan el comportamiento reproductivo normal y respaldan el resultado seronegativo a VPRRS en granjas de selva. Mayor TC en granja positiva a VPRRS en 2016 con respecto a 2015 sugiere desarrollo de procesos adaptativos fisiológicos de la marrana inducidos por la presencia de la enfermedad debido a la inmunoregulación.

Palabras clave: VPRRS, tamaño de camada, gestación

\section{Abstract}

The present study evaluated the duration of days of gestation (DG) and litter size (LS) in pigs of a seropositive central coast farm and seronegative central tropics farm to Porcine Reproductive and Respiratory Syndrome Virus (VPRRS) during the years 2015 and 2016. Sows from VPRRS-positive farm showed increased DGs and LSs decreased in

\footnotetext{
${ }^{1}$ Instituto de Educación Superior Huando, Perú

${ }^{2}$ Universidad Nacional José Faustino Sánchez Carrión, Huacho, Perú

${ }^{3}$ E-mail: mluchocerga@gmail.com; fairahuacho@unjfsc.edu.pe
}

Recibido: 1 de abril de 2019

Aceptado para publicación: 6 de enero de 2020

Publicado: 31 de marzo de 2020 
VPRRS-positive farm compared to those from seronegative tropical farm in 2015. DGs from VPRRS-positive farm pigs were shorter in 2015 compared to 2016. The DG and the LS were not influenced by the time of artificial insemination. Lower DG with higher LS expresses normal reproductive performance and support the seronegative result to VPRRS in tropical farms. Increased LS in positive farm to VPRRS in 2016 with respect to 2015 suggests the development of physiological adaptive processes of sows induced by the presence of the disease due to immunoregulation.

Key words: VPRRS, litter size, gestation

\section{INTRODUCCIÓN}

El síndrome respiratorio y reproductivo porcino (PRRS, por sus siglas en inglés) es una de las enfermedades infecciosas porcinas más importantes desde el punto de vista económico que afecta a la industria porcina a nivel mundial (Jeong et al., 2017). El agente causante es el virus PRRS (VPRRS), que pertenece al orden Nidovirales, familia Arteriviridae, género Arterivirus (Jeong et al., 2017).

En 2012, el SENASA reportó oficialmente la presencia de VPRRS en el Perú (SENASA, 2012). Ramírez et al. (2013) aislaron e identificaron al genotipo 1-europeo del VPRRS en granjas porcinas de las provincias de Lima y Arequipa. En 2014, exámenes serológicos de animales enfermos confirmaron la presencia de VPRRS en una granja limeña, que reportó una disminución del $35.6 \%$ de las utilidades potenciales (Velásquez et al., 2016). Quevedo et al. (2018), por otro lado, identificaron $17.4 \%$ de seroprevalencia del VPRRS en cerdos de crianza no tecnificada en 22 de los 23 departamentos del Perú.

La infección por VPRRS puede ser epidémica y endémica. La infección epidémica se produce cuando huéspedes no inmunizados previamente se infectan independientemente de la edad del individuo, mientras la infección endémica ocurre en subpoblaciones susceptibles con nula o disminuida inmunidad contra el virus (Jeong et al., 2017). La infección por VPRRS se puede dividir en al menos tres etapas: infección aguda, persistencia y extinción; cada una es única en términos de inmunología, virología y signos clínicos. La primera etapa se caracteriza por una infección aguda en pulmones y tracto respiratorio superior, con viremia entre $6 \mathrm{y}$ $12 \mathrm{~h}$ después de la infección. En la segunda etapa, la infección persistente se caracteriza por replicación viral, principalmente, en órganos linfoides, incluidas las amígdalas y los ganglios linfáticos, pero no el bazo (Wills et al., 1997; Rowland et al., 2003), mientras que en la etapa final la replicación del virus decae gradualmente hasta que se extingue en el hospedero (Lunney et al., 2016). No se sabe exactamente cuándo desaparece el virus, pero la replicación se puede mantener hasta 250 días después de la infección(Wills et al., 2003). Sin embargo, se debe tener en cuenta que, en el contexto del entorno típico de producción porcina, el VPRRS establece una infección «de por vida» (Lunney et al., 2016).

Vías directas e indirectas están involucradas en la transmisión de VPRRS entre granjas, tales como la introducción de animales o semen infectado, vehículos de transporte, aerosoles, insectos voladores, aves acuáticas y fómites (Lambert et al., 2012). Los cerdos infectados desarrollan una respuesta humoral fuerte y rápida, pero los anticuerpos iniciales no confieren protección e incluso pueden ser dañinos por mediar un aumento anticuerpo dependiente de la en- 
fermedad (Mateu y Diaz, 2007). La estrategia de control más común para VPRRS endémico sería la vacunación. Jeong et al. (2017) reporta menor tiempo de gestación, mayor número de lechones vivos y destetados en marranas vacunadas contra VPRRS.

Los síntomas clínicos típicos de VPRRS son enfermedad respiratoria leve a grave en cerdos recién nacidos y en crecimiento y fallo reproductivo en cerdas gestantes (Lunney et al., 2016). En marranas preñadas, el VPRRS atraviesa la interfaz materno-fetal infectando fetos con alta eficiencia solo en el tercer trimestre de gestación (Wang et al., 2015), aunque algunas cepas pueden atravesarla durante la etapa media de gestación, matando fetos con moderada eficiencia (Han et al., 2017). Comúnmente, entre el 5 y $80 \%$ de marranas infectadas con VPRRS en fase aguda paren entre el día 100 y 108 de gestación y sus camadas están compuestas de lechones normales, débiles, muertos, en estado autolitico (marrón), y/o fetos momificados (Zimmerman et al., 2019).

El presente trabajo tuvo como objetivo evaluar el comportamiento reproductivo de marranas de granjas seropositivas a VPRRS en la costa peruana en los años 2015/2016 comparado al de marranas de granja seronegativas en condiciones de selva en 2015.

\section{Materiales y Métodos}

La granja seropositiva a VPRRS se encuentra ubicada en la costa central del Perú, distrito de Lurín, provincia de Lima, Perú, 260 msnm. La granja seronegativa se ubica en la selva central del Perú, distrito y provincia de Chanchamayo, departamento de Junín, a $750 \mathrm{msnm}$, con marcadas diferencias de temperatura y humedad relativa entre ambas regiones, El presente estudio se enfocó en analizar el efecto de la presencia de VPRRS sobre el rendimiento reproductivo de la marrana.
Se evaluó el registro reproductivo de 1020 marranas de la línea genética Camborough 2, correspondientes al total de la población de las granjas evaluadas (granja de selva seronegativa a VPRRS: 247; granja seropositiva de la costa central de 2015 y 2016: 442 y 331 registros, respectivamente. Ambas granjas se encuentran dentro del programa de detección de VPRRS realizado por el Servicio Nacional de Sanidad Agraria (SENASA) y los resultados de seropositividad de las dos granjas corresponden a dos muestreos en 2015. Los muestreos contra VPRRS en ambas granjas fue realizado en julio y diciembre de 2015 y 2016 . Los animales no son vacunados contra VPRRS, toda vez que a la fecha (diciembre de 2019), el SENASA no ha autorizado el uso de vacunas contra esta enfermedad.

Las granjas evaluadas emplean la inseminación artificial como método reproductivo. En la granja de costa seropositiva a VPRRS, se utilizó semen externo (Atahuampa PIC) inmediatamente después de detectado el virus. La granja de selva seronegativa utiliza semen de sus propios verracos. Ambas granjas aplican el sistema de manejo «todo dentro/todo fuera» en sus actividades (reemplazo de reproductoras, inseminación artificial, partos, destete, engorde). Asimismo, la selección de sus reproductoras es en función a la velocidad de crecimiento, aplomos, número y forma de pezones y estado sanitario sin presencia de enfermedades infectocontagiosas; mientras que el descarte se realiza por la edad (no más de siete partos), baja prolificidad, abortos, enfermedades infectocontagiosas y problemas podales.

Los datos reproductivos corresponden a marranas de uno a ocho partos. El tamaño de camada consideró el número total de lechones nacidos, incluyendo momificados y mortinatos. Para evaluar el efecto climático, los datos reproductivos fueron denominados temporada fría y temporada caliente y se relacionaron con el día de la inseminación artificial. Para el análisis estadístico se utilizó la prueba no paramétrica de Kruskal-Wallis con el programa $\mathrm{R}$. 

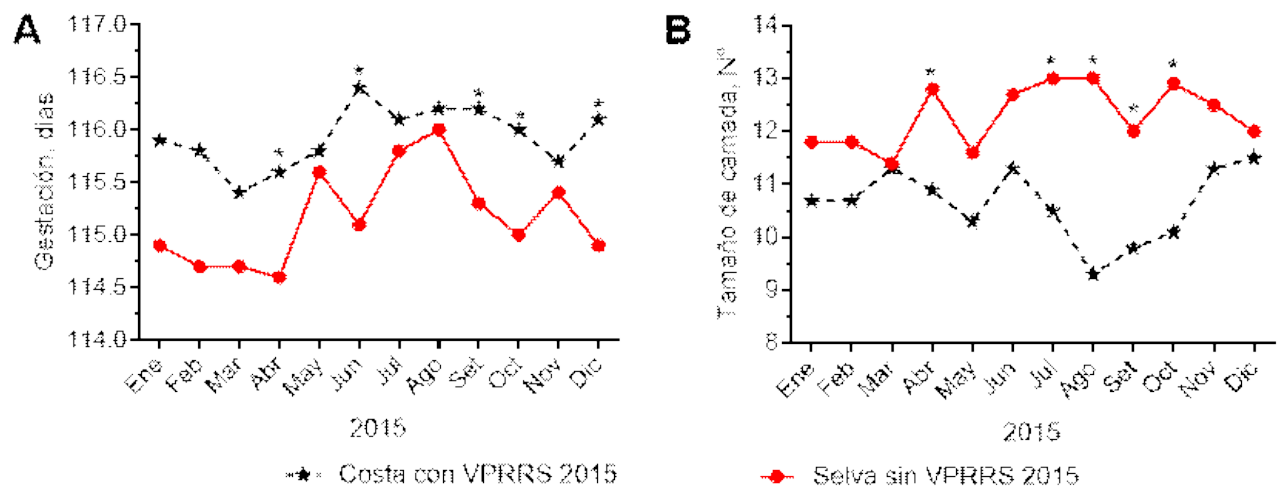

Figura 1. Tiempo de gestación (A) y tamaño de camada (B) en una granja de selva central libre del virus del Síndrome Reproductivo Respiratorio Porcino (VPRRS) y en una granja de costa central positivo a VPRRS durante el año 2015
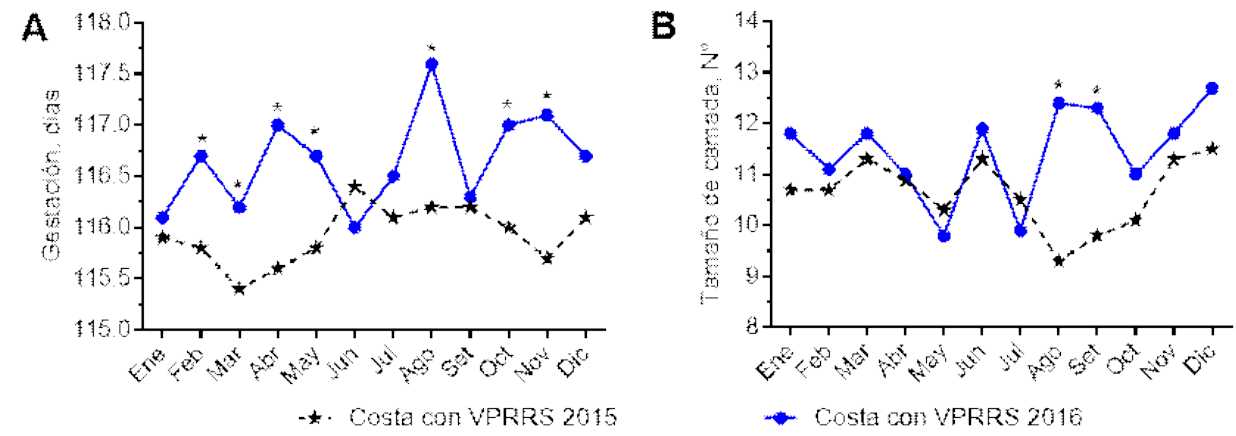

Figura 2. Comportamiento reproductivo de marranas de granja seropositivas al virus del Síndrome Reproductivo Respiratorio Porcino (VPRRS) durante 2015 y 2016

\section{Resultados}

El tiempo de gestación (DG) de las marranas de granja de costa seropositivas a VPRRS fue significativamente mayor durante los meses de enero, abril, junio, setiembre, octubre y diciembre $(\mathrm{p}<0.05)$ comparado con el de las marranas de selva seronegativas durante 2015. Asimismo, la tendencia de mayor DG en marranas de granjas seropositivas a VPRRS coincidieron con tendencias de tamaño de camada (TC) disminuida comparado con marranas de selva seronegativas durante el año 2015, observándose diferencias estadísticas de TC en los meses de abril, julio, agosto, setiembre y octubre $(\mathrm{p}<0.05)$ (Figura 1).
Los DG de marranas de granjas seropositivas a VPRRS durante el año 2015 (primer año en condición seropositiva) fueron menores en comparación con el año 2016, donde las marranas muestran mayores DG, con diferencias significativas en los meses de febrero, marzo, abril, mayo, agosto, octubre y noviembre $(p<0.05)$. Sin embargo, los DG disminuidos no coinciden con mayores TC en 2015, ni los mayores DG de 2016 coinciden con menor TC, con excepción de los meses de agosto y setiembre $(\mathrm{p}<0.05)$ (Figura 2$)$.

Si bien la temporada de inseminación se considera como factor influyente en el comportamiento reproductivo de la marrana, no se encontró diferencias estadísticas para DG y TC entre la temporada fría y caliente, ni 

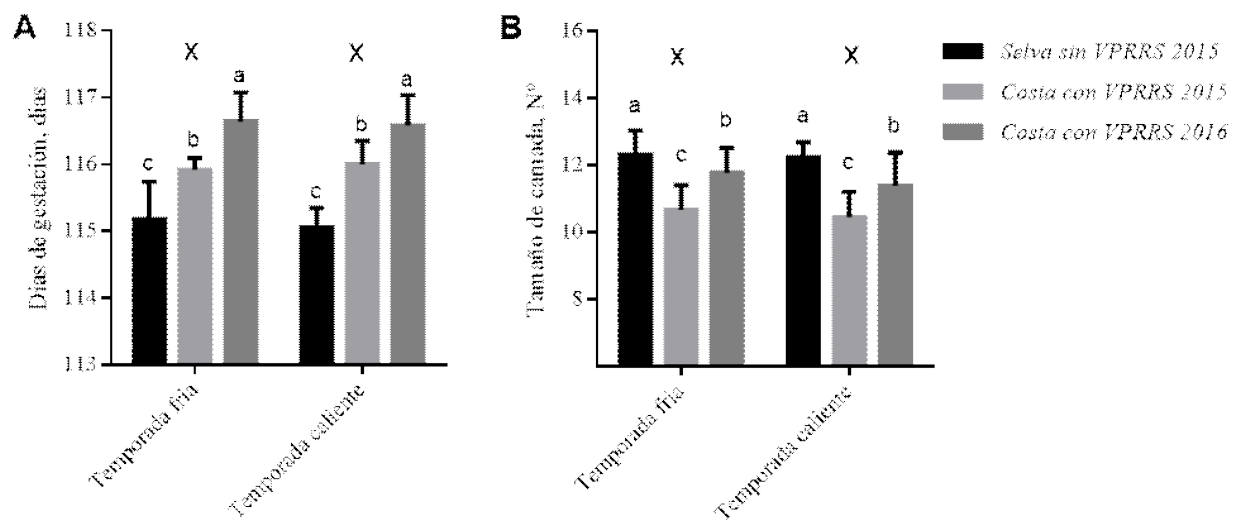

Figura 3. Efecto de la estación de inseminación sobre los días de gestación (A) y tamaño de camada (B) en granjas porcinas positivas y negativas al virus del Síndrome Reproductivo Respiratorio Porcino (VPRRS)

correlación significativa entre estas dos variables. Marranas de granja de selva seronegativas a VPRRS muestran los menores DG y los mayores TC en ambas estaciones $(\mathrm{p}<0.05)$. Al analizar el comportamiento reproductivo de marranas de granja de costa seropositivas a VPRRS, los mayores DG y TC se observan en ambas temporadas en el año 2016 comparado con el comportamiento del año $2015(\mathrm{p}<0.05)$ (Figura 3).

\section{Discusión}

El VPRRS es de las enfermedades de mayor repercusión económica para los porcicultores (Kleiboeker et al., 2005), ocasionando pérdidas económicas significativas debido al brote infeccioso y a su permanencia endémica, con posibilidades de reemerger como brote agudo (Benfield et al., 1999). Si bien, las pérdidas por la forma endémica son leves, los índices de fertilidad y ganancia de peso disminuidos atentan con la rentabilidad porcina (Lopez-Heydeck et al., 2015).

En el presente estudio, marranas provenientes de granja de costa seropositivas a VPRRS tuvieron mayor duración de la gestación y un menor TC, mientras que la menor duración de la gestación de marranas de granja de selva seronegativos a VPRRS se reflejó con mayores TC. Estos resultados se explicarían en el hecho que granjas seropositivas a VPRRS reportan mayor número de fetos momificados, mortinatos y nacidos débiles, así como mayores casos de abortos en la etapa final de la gestación (Benfield et al., 1999; Zimmerman et al., 2012) y que conllevan a un aumento de los DG y disminución del TC. Los datos clínicos mostraron una mejora significativa en el rendimiento reproductivo de marranas vacunadas en granjas endémicas a VPRRS. Asimismo, Olanratmanee et al. (2014), al evaluar el rendimiento reproductivo de marranas en hatos con VPRRS seropositivo con y sin vacunación con virus vivos modificados con PRRS, encontraron un menor rendimiento reproductivo en marranas no vacunadas.

Marranas nulíparas procedentes de una explotación libre de VPRRS mostraron notable reducción de fertilidad al ser introducidas sin vacunación en granjas de baja prevalencia a VPRRS que no realizaban reposición externa por más de un año (Bernal y Callen, 2003). El parto prematuro es uno de los síntomas de VPRRS durante el último tercio de la gestación (Zimmerman et al., 2012), y se 
caracteriza por el incremento de muertes intraparto y nacimientos de lechones de bajo peso con apnea y alteraciones metabólicas graves (Mota-Rojas et al., 2015). Los casos de parto prematuro pueden reducirse por efecto de vacunación de la marrana con VPRRS, observándose aumento en la duración de la gestación y reducción en el número de lechones nacidos muertos (Jeong et al., 2017). En el presente estudio, el síntoma de días de gestación aumentado fue observado en marranas de granjas de costa seropositivas durante 2015, pero con tendencia a la normalización durante 2016, lo que sugiere que las marranas desarrollaron inmunidad pasiva contra el VPRRS recuperando su productividad.

La inmunosupresión que ocasiona el VPRRS genera una enfermedad con inmunidad de instauración lenta que permite prolongados tiempos de viremia que favorece su diseminación (Benfield et al., 1999; Xiao et $a l ., 2010)$. Está peculiar respuesta inmune, sumado a la variabilidad genética, generan grupos de animales con desafíos diferentes: animales no infectados, en proceso de infección y excreción vírica, animales recuperados de infección y que están protegidos, animales recuperados de infección en fase de pérdida de protección y que vuelven a ser susceptibles, así como animales portadores (Benfield et al., 1999; Lopez-Heydeck et al., 2015).

La estación, debido a diferencias de temperatura, humedad relativa y fotoperiodo, influyen sobre las variables reproductivas de la cerda (Galé et al., 2015); sin embargo, en el presente estudio no se encontró diferencia significativa en el comportamiento reproductivo de las cerdas entre la temporada fría y la caliente. La temperatura ambiental que enfrenta una granja de selva durante todo el año es superior a la de una granja de costa. La aparente mayor duración de días de gestación en la granja de costa seropositiva estaría siendo encubierto por la mayor duración de días de gestación que mostrarían las marranas criadas en condiciones ambientales de selva. El estrés calórico que enfrentan marranas durante la temporada caliente influye negativamente sobre la síntesis de progesterona placentaria disminuyendo su nivel sanguíneo (Knight y Kukoly, 1990)

Tretipskul et al. (2012) observaron efectos negativos en la producción de semen en machos reproductores en temporadas de alta humedad relativa y de lluvia. Se debe considerar que el parto en las marranas ocurre por efecto de la inseminación con semen obtenido en la estación anterior; así, la inseminación realizada en el verano muestra los menores índices reproductivos en todo el periodo de lluvias; mientras que la inseminación realizada a mediados y fin de la temporada fría se relacionan con un aumento del total de lechones nacidos durante la temporada caliente (Suriyasomboon et al., 2006). Los índices de fertilidad en regiones tropicales pueden estar influenciados por la calidad del semen, sin embargo, se debe de tener en cuenta que pobres índices de fertilidad podrían estar afectados por factores como la función ovárica, el estrés resultante del aborto o el manejo (Tretipskul et al., 2012).

La presencia de VPRRS en granjas porcinas altera el comportamiento reproductivo de las marranas. Si bien en el presente estudio, el parto prematuro no se visualiza como un síntoma de la presencia de VPRRS, los mayores DG de gestación de marranas seropositivas coincidieron con sus menores TC.

\section{Literatura Citada}

\section{Benfield D, Collins J, Dee S, Halbur} P, Joo H, Lager K, et al. 1999. Porcine reproductive and respiratory syndrome. In: Straw BE, D'Allaire S, Mengeling WL, Taylor DJ (eds). Diseases of the swine. $8^{\text {th }}$ ed. Ames, Iowa: Iowa State University Press. p. 201-232. 
2. Bernal R, Callen A. 2003. Reproductive impact of introduction of negative nulliparous gilts against PRRS, vaccinated or not with inactivated vaccine (Progressis), in positive farms with low prevalence. In: Conference of Veterinary Medicine. Tartu, Estonia.

3. Galé I, Izaguirre M., Grandía J, Daza A. 2015. Efecto de la estación de cubrición sobre los resultados reproductivos de cerdas en Aragón. En: XVI Jornadas de Producción Animal AIDA. Zaragoza-España.

4. Han J, Zhou L, Ge X, Guo X, Yang H. 2017. Pathogenesis and control of the Chinese highly pathogenic porcine reproductive and respiratory syndrome virus. Vet Microbiol 209: 30-47. doi: 10.1016/j.vetmic.2017.02.020

5. Jeong J, Kim S, Park KH, Kang I, Park SJ, Park C, Chae C. 2017. Evaluation of the effect of a porcine reproductive and respiratory syndrome (PRRS) modified-live virus vaccine on sow reproductive performance in endemic PRRS farms. Vet Microbiol 208: 47-52. doi: 10.1016/j.vetmic.2017.07.016

6. Kleiboeker SB, Schommer SK, Lee SM, Watkins S, Chittick W, Polson D. 2005. Simultaneous detection of North American and European porcine reproductive and respiratory syndrome virus using real-time quantitative reverse transcriptase-PCR. Brief communication. J Vet Diagn Invest 17: 165-170. doi: 10.1177/104063870501700211

7. Knight JW, Kukoly CA. 1990. In vitro release of progesterone and estrone by the porcine placenta throughout gestation. Domest Anim Endocrinol 7: 497-508. doi: 10.1016/0739-7240(90)90007-m

8. Lambert MÈ, Poljak Z, Arsenault J, D'Allaire S. 2012. Epidemiological investigations in regard to porcine reproductive and respiratory syndrome (PRRS) in Quebec, Canada. Part 1: biosecurity practices and their geographical distribution in two areas of different swine density. Prev Vet Med 104: 7483. doi: 10.1016/j.prevetmed.2011.12.004

9. López-Heydeck S, Alonso-Morales R, Mendieta-Zerón H, Vázquez Chagoyán J. 2015. Síndrome reproductivo y respiratorio del cerdo (PRRS): revisión. Rev Mex Cienc Pecu 6: 69-89.

10. Lunney JK, Fang Y, Ladinig A, Chen $N$, Li Y, Rowland B, Renukaradhya GJ. 2016. Porcine Reproductive and Respiratory Syndrome Virus (PRRSV): pathogenesis and interaction with the immune system. Annu Rev Anim Biosci 4: 129-154. doi: 10.1146/annurev-animal022114-111025

11. Mateu E, Diaz I. 2008. The challenge of PRRS immunology. Vet J 177: 345 351. doi: 10.1016/j.tvj1.2007.05.022

12. Mota-Rojas D, Fierro R, Roldan-Santiago P, Orozco-Gregorio H, GonzálezLozano M, Bonilla H, MartínezRodríguez R, et al. 2015. Outcomes of gestation length in relation to farrowing performance in sows and daily weight gain and metabolic profiles in piglets. Anim Prod Sci 55: 93. doi: 10.1071/an13175

13. Olanratmanee EO, Thanawongnuwech $R$, Kunavongkrit A, Tummaruk $P$. 2014. Reproductive performance of sows with and without PRRS modified live virus vaccination in PRRSvirus-seropositive herds. Trop Anim Health Pro 46: 1001. doi: 10.1007/ s11250-014-0606-5.

14. Quevedo M, Mantilla J, Portilla K, Villacaqui R, Rivera H. 2018. Seroprevalencia del virus del Síndrome Reproductivo y Respiratorio Porcino en cerdos de crianza no tecnificada del Perú. Rev Inv Vet Perú 29: 643-651. doi: 10.15381/rivep.v29i2.14497

15. Ramirez M, Rivera H, Manchego A, More J, Chiok KL. 2013. Aislamiento y genotipificación virus del síndrome respiratorio y reproductivo porcino (VPRRS) en granjas seropositivas de las provincias 
de Lima y Arequipa, Perú. Rev Inv Vet Perú 24: 222-232. doi: 10.15381/ rivep.v24i2.2512

16. Rowland RR, Lawson S, Rossow K, Benfield DA. 2003. Lymphoid tissue tropism of porcine reproductive and respiratory syndrome virus replication during persistent infection of pigs originally exposed to virus in utero. Vet Microbiol 96: 219-235. doi: 10.1016/ j.vetmic.2003.07.006

17. [SENASA] Servicio Nacional de Sanidad Agraria. 2012. Perú notifica presencia virus del PRRS en una explotación porcina. [Internet]. Disponible en: https://www.3tres3.com/ultima-hora/ peru-notifica-presencia-del-virusdelprrs-en-una-explotacionporcina_30671/

18. Suriyasomboon A, Lundeheim $N$, Kunavongkrit A, Einarsson S. 2006. Effect of temperature and humidity on reproductive performance of crossbred sows in Thailand. Theriogenology 65 : 606-628. doi: $10.1016 / \mathrm{j}$.theriogenology.2005.06.005

19. Tretipskul C, Am-in N, Tummaruk P, Techakumphu M. 2012. Season and breed effects on sperm production in PRRS free boars. Thai J Vet Med 42: 471-476.

20. Velásquez C, Vega J, Cerga ML. 2016. Síndrome Reproductivo Respiratorio Porcino: presentación en el tiempo y efecto sobre los parámetros productivos y reproductivos. Rev Inv Vet Perú 27: 813-821. doi: 10.15381/rivep.v27i4.12569

21. Wang G, Yu Y, Tu Y, Tong J, Liu Y, Zhang C, Chang Y, et al. 2015. Highly pathogenic porcine reproductive and respiratory syndrome virus infection induced apoptosis and autophagy in thymi of infected piglets. PLoS ONE 10(6): e0128292. doi: 10.1371/ journal.pone.0128292

22. Wills RW, Doster AR, Galeota JA, Sur JH, Osorio FA. 2003. Duration of infection and proportion of pigs persistently infected with porcine reproductive and respiratory syndrome virus. J Clin Microbiol 41: 58-62. doi: $10.1128 / \mathrm{jcm} .41 .1 .58-62.2003$

23. Wills RW, Zimmerman JJ, Yoon KJ, Swenson SL, McGinley MJ, Hill HT, Platt KB, et al. 1997. Porcine reproductive and respiratory syndrome virus: a persistent infection. Vet Microbiol 55: 231-240. doi: 10.1016/s0378-1135(96)01337-5

24. Xiao S, Jia J, Mo D, Wang Q, Qin L, He Z, Zhao X, Huang Y, Li A, Yu J, Niu Y, Liu X, Chen Y. 2010. Understanding PRRSV infection in porcine lung based on genome-wide transcriptome response identified by Deep sequencing. Plos One 5: e11377. doi: 10.1371/journal.pone.0011377

25. Zimmerman JJ, Benfield DA, Dee SA, Murtaugh MP, Stadejek T, Stenvenson GW, Torremorell M. 2012. Porcine reproductive and respiratory syndrome virus (porcine arterivirus). In: Zimmerman JJ, Karriker LA, Ramirez A, Schwartz KJ, Stevenson GW (eds). Diseases of swine. $10^{\text {th }}$ ed. Ames, USA: Wiley-Blackwell. p 461-486.

26. Zimmerman J, Scott D, Holtkamp D, Murtaugh M, Stadejek T, Stevenson G, Torremorell M, et al. 2019. Porcine reproductive and respiratory syndrome viruses (porcine Arteriviruses). In: Zimmerman J, et al. (eds). Diseases of swine, $11^{\text {th }}$ ed. John Wiley. p 685-708. 